\title{
Étude des ferments lactiques des levains naturels de Normandie sélectionnés par une inhibition des Gram-négatifs
}

\author{
par
}

\author{
J. Jacquet, O. Coiffier et P. Hélaine*
}

\section{INTRODUCTION}

Dans la fabrication du fromage de Camembert, un certain nombre d'industriels préfèrent, encore actuellement, utiliser leurs propres levains élaborés à partir des laits crus de leurs meilleurs fournisseurs, plutôt que ceux qu'ils pourraient se procurer dans le commerce. Ils considèrent, en effet, ces derniers comme trop uniformes et « passepartout ", peut-être même, mal adaptés à leurs conditions locales. Ils espèrent, aussi, en se servant des microbes de leur environnement, conserver une "personnalité ", un "cru ", à leurs produits, dans la mesure où celui-ci dépend de l'action de l'ensemencement lactique venant se surajouter à la composition initiale du lait récolté. Malgré le faible pourcentage de Camemberts fabriqués avec du lait cru, à l'époque où la recherche du naturel par le consommateur constitue une mode grandissante, c'est une question qui risque de prendre de l'actualité et qui mérite attention.

Nous avons voulu connaître la composition spécifique de ces levains, afin d'en mesurer l'intérêt, et éventuellement, améliorer les conditions de fabrication. Ce sera, aussi, l'occasion d'approfondir nos connaissances sur les ferments lactiques qui peuplent les laits crus, dont, à vrai dire, on sait bien peu de choses, si ce n'est une assez grande fréquence de résistance "spontanée " aux antibiotiques, comme l'un de nous l'a observé avec Mlle Auxepaules [6].

\footnotetext{
* Laboratoire de Microbiologie de l'Université de Caen - 14032 Caen cedex.
} 


\section{PREPARATION DES LEVAINS ET ISOLEMENT DES SOUCHES}

Quatre levains ont été obtenus en enrichissant progressivement en ferments lactiques, par inhibition des Gram-négatifs présents, des laits de mélange provenant de quatre laiteries réputées pour la fabrication de Camemberts traditionnels**.

Les échantillons ont été additionnés d'acide nalidixique, qui empêche le développement des bactéries Gram-négatives, à la concentration de $50 \mu \mathrm{g}$ par $\mathrm{ml}$, puis soumis à des repiquages successifs jusqu'à disparition des coliformes [3].

Dans ces levains, la population mésophile totale obtenue par cette sélection a été comprise entre 6 et $24.10^{8}$ micro-organismes par $\mathrm{ml}$, sur milieu plate count agar. La flore très largement dominante était constituée par des streptocoques lactiques. Pour en préciser la nature, des colonies ont été prélevées sur les boîtes de numération en nombre égal au nombre entier le plus proche de la racine carrée du nombre total des colonies dénombrées.

Nous en avons obtenu 69 qui, reprises en milieu liquide de Hogg et Jago [5], ont été ensuite isolées sur milieu solide de Reddy [12] puis repiquées.

\section{IDENTIFICATION BIOCHIMIQUE ET CLASSIFICATION DES SOUCHES}

L'identification des souches a été effectuée selon le procédé Api System Lactobacillus [11] permettant l'étude de 49 caractères biochimiques et qui est applicable également aux streptocoques.

Les recherches effectuées avaient trait :

- aux substrats susceptibles d'être fermentés avec production d'acide (glycérol, érythritol, d(-)arabinose, $1(+)$ arabinose, ribose, $\mathrm{d}(+)$ xylose, $1(-)$ xylose, adonitol, méthyl-xyloside, galactose, $d(+)$ glucose, $\mathrm{d}(-)$ lévulose, $\mathrm{d}(+)$ mannose, $\mathrm{l}(-)$ sorbose, rhamnose, dulcitol, mésoinositol, mannitol, sorbitol, méthyl-d-mannoside, méthyl-d-glucoside, $\mathrm{N}$-acétyl-glucosamine, amygdaline, arbutine, esculine, salicine, d(+) cellobiose, maltose, lactose, $\mathrm{d}(+)$ mélibiose, saccharose, $\mathrm{d}(+)$ tréhalose, inuline, $\mathrm{d}(+)$ mélézitose, $\mathrm{d}(+)$ raffinose, dextrine, amylose, amidon, glycogène, gluconate de sodium) ;

** Nous remercions $M$. Bailleul, Directeur du Laboratoire Interprofessionnel laitier du Calvados pour sa collaboration dans la préparation des levains. 
- à l'apparition, ou non, de gaz lors de la fermentation du glucose ;

- à l'existence d'enzymes bactériennes (uréase, $\beta$-galactosidase, catalase, nitrate-réductase) ;

- à la formation d'un métabolite terminal de la fermentation (acétoïne) ;

- à certains inhibiteurs possibles (citrate de sodium, teepol à 4 p. 100 et à 6 p. 100).

\section{TABLEAU 1}

Répartition des espèces identifiées dans les levains étudiés

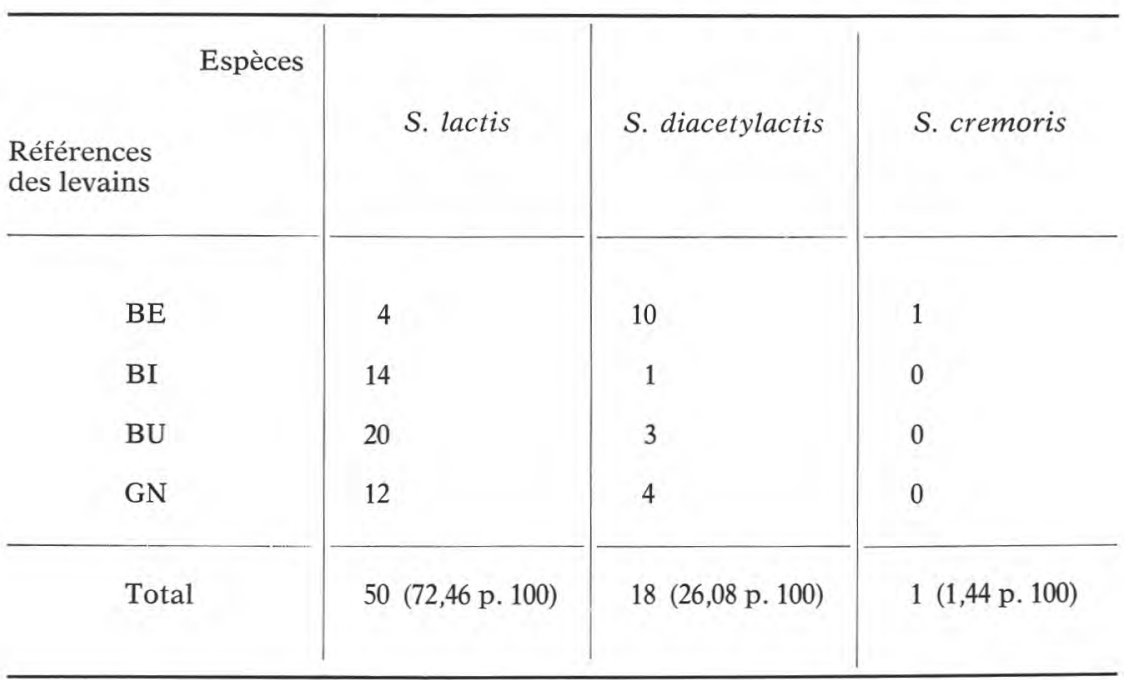

Nos résultats de détermination des 69 souches que nous avons isolées des levains artisanaux normands figurent dans le tableau 1.

Cette étude effectuée avec un matériel normalisé, nous a permis de réaliser une classification adansonienne des différents clones isolés [1].

Pour établir le diagramme qui fait l'objet de la figure 1, représentant la matrice des coefficients de similitude entre les souches de $S$. lactis, $S$. diacetylactis et $S$. cremoris obtenues, nous n'avons retenu que les aptitudes à transformer, ou non, les différents substrats, san tenir compte de la rapidité d'apparition des caractères qui permet d'établir, par ailleurs, le profil de la souche à identifier. Nous pouvons, ainsi, mettre en évidence six taxons. Dans chacun d'eux, nous constatons l'existence de deux, ou plusieurs, souches identiques par leurs caractères biochimiques, mais différant par leur origine ou leur 


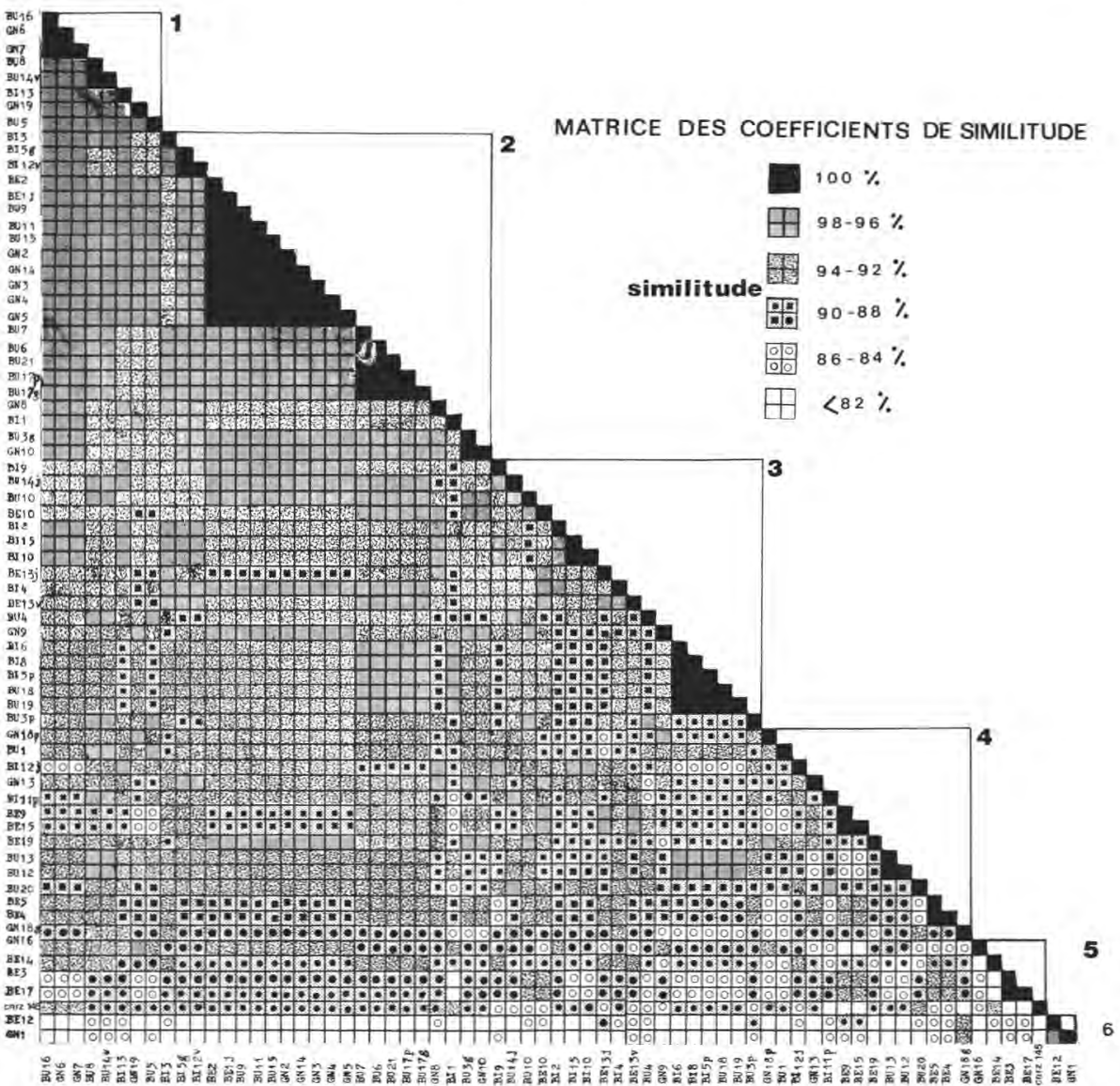

fig. 1

Diagramme de la classification adansonienne des souches de Streptococcus lactis et $S$. diacetylactis des levains naturels de quatre usines de Normandie obtenus après action de l'acide nalidixique, Le premier taxon comprend huit $S$. lactis extrêmement proches (96 à 100 p. 100 de similitude) quoique provenant de trois établissements différents. En revanche, trois taxons renferment des souches présentes dans chacun des levains. L'un de ceux-ci est tout à fait remarquable par la variété des bactéries qu'il renferme et qui se répartissent dans les six taxons.

pouvoir acidifiant. Le premier taxon comprend huit Streptococcus lactis très proches les uns des autres ( 96 p. 100 à 100 p. 100 de similitude), quoique issus de trois levains différents. Les trois taxons suivants sont tous composés de souches présentes dans les quatre levains. Autrement dit, chacun de ceux-ci réunit toute une variété de streptocoques. Enfin, le sixième taxon ne comprend que deux souches. 
Du point de vue de la diversité des chimiotypes, le levain G N est le plus remarquable ; il serait le meilleur pour ceux qui estiment cette variété comme favorable pour la production de l'arôme, les échanges de facteurs de croissance entre souches, et surtout la résistance aux phages toujours présents dans les laits crus : il renferme, en effet, des souches réparties dans les six taxons.

Aucun des clones isolés par nous ne se rapproche à plus de 94 p. 100 de la souche $S$. lactis, CNRZ 145 prise comme référence et qui se classe dans le cinquième taxon.

\section{COURBES D'ACIDIFICATION}

Le pouvoir acidifiant des souches a été testé en lait UHT autoclavé $30 \mathrm{~min}$ à $100^{\circ} \mathrm{C}$, puis ensemencé à $1 \mathrm{p}$. 100 à partir d'une culture en bouillon de Man, Rogosa et Sharpe [9].

L'acidité, mesurée en degrés Dornic, a été évaluée au bout de 2 h 30,4 h, 5 h 30, 7 h, 8 h, ce qui a permis d'établir les courbes d'acidification représentées dans la figure 2.

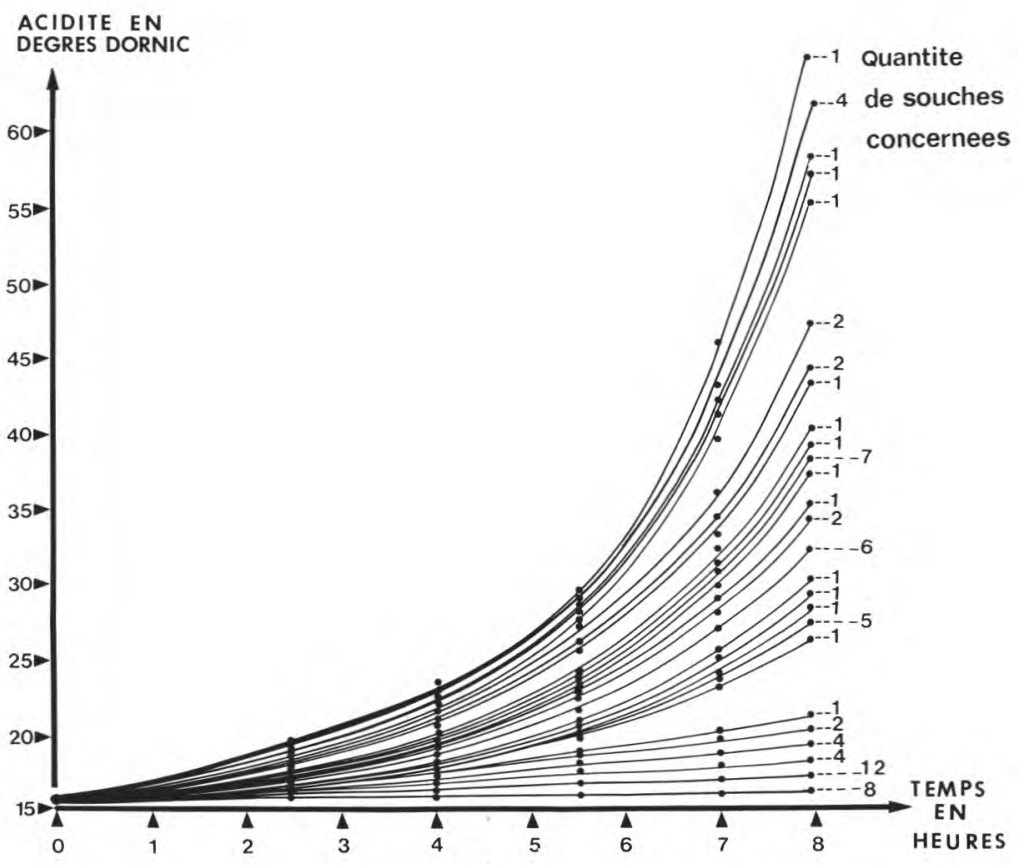

fig. 2

Courbes d'acidification en $8 \mathrm{~h}$ à $30^{\circ} \mathrm{C}$ sur lait U.H.T. des différentes souches de streptocoques isolés des levains étudiés. 
Nous pouvons ainsi remarquer que les espèces présentes dans les levains naturels, très diversifiées dans leurs chimiotypes, le sont, aussi, par leur action sur le lactose. Par ailleurs, nous avons noté que des souches présentant des profils identiques pour les cinquante caractères étudiés, pouvaient avoir des comportements différents dans leur aptitude à la coagulation du lait. C'est le cas, par exemple, dans le premier taxon, des cultures $\mathrm{BU}_{8}$ et $\mathrm{BU} 14 \mathrm{~V}$ dont les acidités produites au bout de 8 h à $30^{\circ} \mathrm{C}$, sont respectivement de $17^{\circ} \mathrm{D}$ et $38^{\circ} \mathrm{D}$.

Nous avons consigné, dans le tableau 2, la répartition des diverses souches étudiées, en fonction de l'acidité obtenue après 8 h d'incubation à $30^{\circ} \mathrm{C}$.

\section{TABLEAU 2}

Répartition des souches étudiées en fonction de l'acidité obtenue après $8 \mathrm{~h}$ d'incubation à $30^{\circ} \mathrm{C}$

\begin{tabular}{c|c|c|c}
\hline & & \\
$\begin{array}{c}\text { Références } \\
\text { des levains }\end{array}$ & $\begin{array}{c}\text { Acidité } \\
\text { égale à } 50^{\circ} \mathrm{D}\end{array}$ & $\begin{array}{c}\text { Comprise entre } \\
30^{\circ} \text { et } 50^{\circ} \mathrm{D}\end{array}$ & $\begin{array}{c}\text { Inférieure } \\
\text { à } 30^{\circ} \mathrm{D}\end{array}$ \\
\hline $\mathrm{BE}$ & 3 & 7 & 4 \\
$\mathrm{BI}$ & 1 & 5 & 9 \\
$\mathrm{BU}$ & 0 & 7 & 16 \\
$\mathrm{GN}$ & 4 & 3 & 9 \\
\hline
\end{tabular}

A l'exception de l'échantillon BU, chaque levain comporte un éventail de ferments ayant des pouvoirs acidifiants très différents et, a priori, les levains $\mathrm{BE}$ et $\mathrm{GN}$, semblent plus équilibrés que les levains $\mathrm{BI}$ et BU. Mais, des essais pratiques en fromagerie seraient nécessaires pour confirmer ce que l'on peut supposer des aptitudes technologiques des micro-organismes, uniquement d'après des expériences de laboratoire.

\section{DENOMBREMENT DE LA FLORE LACTIQUE APRES CULTURE}

Une souche peut être d'autant plus intéressante dans la fabrication de camemberts crus qu'elle occupe rapidement le terrain, afin 
d'entrer en compétition avec d'autres micro-organismes qui pourraient être indésirables, ou moins utiles qu'elle. L'étude de la population lactique a été effectuée sur une fraction de l'échantillon destiné aux mesures d'acidité, congelé après 7 h d'incubation [4].

Nous avons fait figurer, dans le tableau 3, la moyenne arithmétique des dénombrements réalisés avec les différentes souches composant chaque levain. Dans chacun de ceux-ci, une proportion plus ou moins importante de clones atteint des taux supérieurs à $10^{9}$ cellules par $\mathrm{ml}$, au bout de $7 \mathrm{~h}$ à $30^{\circ} \mathrm{C}$.

\section{TABLEAU 3}

Taux bactériens obtenus au bout de $7 \mathrm{~h}$ de culture en lait UHT à $30^{\circ} \mathrm{C}$

\begin{tabular}{|c|c|c|c|c|c|c|c|c|}
\hline Références des & \multicolumn{2}{|c|}{ BI } & \multicolumn{2}{|c|}{$\mathrm{BU}$} & \multicolumn{2}{|c|}{$\mathrm{BE}$} & \multicolumn{2}{|c|}{ GN } \\
\hline Proportion des souches & $50 \%$ & $50 \%$ & $66 \%$ & $33 \%$ & $33 \%$ & $66 \%$ & $60 \%$ & $40 \%$ \\
\hline $\begin{array}{l}\text { Moyenne des numéra- } \\
\text { tions par ml en millions }\end{array}$ & 350 & 1300 & 250 & 950 & 550 & 1400 & 250 & 2550 \\
\hline Valeurs extrêmes & 300 & 2200 & 40 & 1400 & 100 & 1900 & 150 & 5400 \\
\hline
\end{tabular}

Il faut noter qu'il ne s'agissait dans cette étude que de comparer des levains entre eux, et après un temps court, et que nous aurions probablement obtenu des taux cellulaires plus grands avec un autre milieu que le lait UHT.

Bien que, d'après Martley, des populations élevées de streptocoques dans le caillé puissent aboutir quand même, à l'obtention de fromages médiocres [10], des souches très proliférantes peuvent être intéressantes dans la mesure où elles seraient moins affectées par une infection phagique.

\section{SENSIBILITE DES SOUCHES AUX ANTIBIOTIQUES}

La présence d'antibiotiques dans le lait inhibant le développement des bactéries lactiques, entraîne un ralentissement de l'acidification en cours de fabrication, ce qui peut aboutir à l'envahissement par des micro-organismes capables de provoquer des gonflements importants, 
en cours de moulage. Dans les cas extrêmes, le caillé de 24 h prend un aspect de champignon mousseux, comme le montrent la figure 3 d'un accident encore observé récemment, malgré l'ancienneté (1951) et le nombre ultérieur de travaux publiés sur cette question.

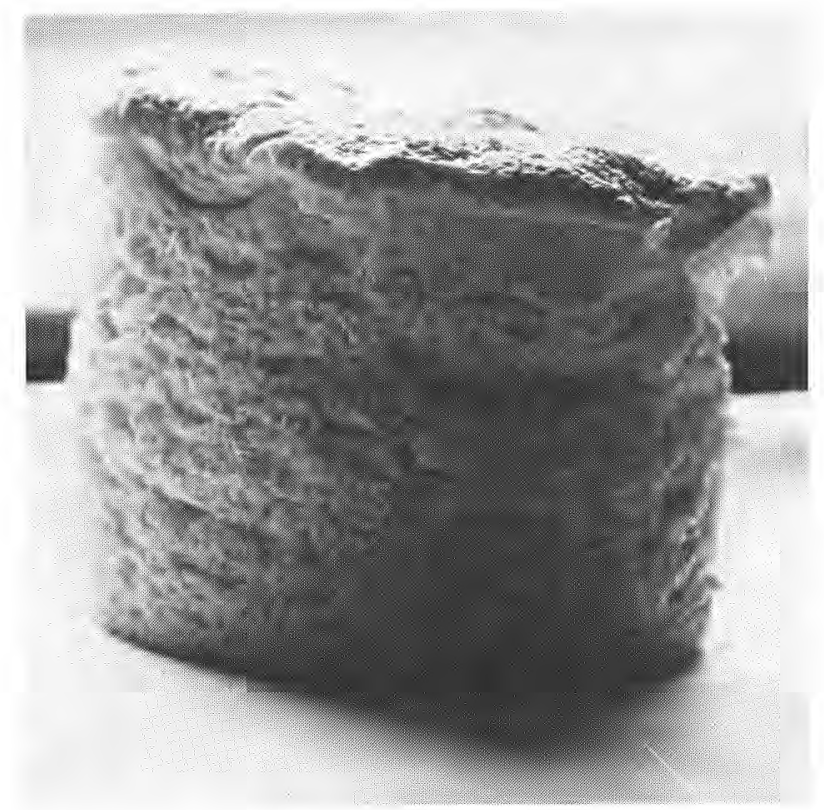

fig. 3

Accident encore provoqué en 1979 sur des Camemberts par la présence dans le lait d'antibiotiques inhibiteurs des ferments lactiques.

Il y avait inhibition pratiquement totale des ferments lactiques et les germes incriminés étaient des coliformes, qui atteignaient des taux de 10000000 par gramme dans le caillé, à partir d'une contamination initiale dans le lait emprésuré de 140000 par ml. Les espèces isolées étaient du genre Enterobacter aerogenes, Klebsiella pneumoniae, Escherichia coli, avec une majorité d'Enterobacter aerogenes (70 p. 100).

Ceci nous aménerait tout naturellement à essayer de sélectionner des ferments lactiques capables de se multiplier, malgré la présence d'antibactériens bien que, du point de vue de l'hygiéniste, il semble difficile d'admettre la fabrication de fromages contenant de telles substances. Cependant, il était intéressant de connaître le comporte- 
ment des souches " sauvages » isolées, à l'égard d'un certain nombre d'antibiotiques, tels que la pénicilline $(\mathrm{P})$, l'oxacilline $(\mathrm{OX})$, la streptomycine (S), la gentamicine (GM), l'auréomycine (A), la tétracycline (TE), le chloramphénicol (C), les sulfamides (SU).

Les antibiogrammes ont été réalisés sur gélose trypticase soja à partir d'une culture en bouillon Brain Heart. Dans le tableau 4, figurent les antibiotypes ainsi que le nombre de souches concernées par chacun de ceux-ci. La presque totalité (98 p. 100) de ces streptocoques est résistante aux sulfamides. Dans l'ensemble de la population étudiée, on note l'existence d'un seul Streptococcus lactis multirésistant, et deux souches résistant à trois antibiotiques à la fois.

\section{TABLEAU 4}

Antibiotypes des souches étudiées

\begin{tabular}{l|c}
\hline \multicolumn{1}{c|}{ Antibiotypes } & Nombre de souches \\
\hline OX.S.GM.A.T.C.SU. & 1 \\
A.T.SU. & 1 \\
P.S.SU. & 1 \\
P.SU. & 5 \\
S.SU. & 1 \\
P. & 1 \\
SU. & 52 \\
\end{tabular}

\section{CONCLUSION}

Ce bref tour d'horizon des principales propriétés de quelques levains artisanaux nous a montré la très grande diversité des souches sauvages qui les composent, avec la prédominance de Streptococcus lactis, ce qui avait été déjà souligné dans l'ouvrage sur le lait et le froid [7], il y a 20 ans.

La diversité naturelle constitue un mélange d'espèces, qui sont, les unes, très acidifiantes, les autres douées d'un pouvoir de multiplication élevé, sans compter la fourniture de métabolites divers. C'est sans doute grâce à cet éventail d'individus qu'il existe une certaine 
" harmonie » entre les streptocoques lactiques et les populations phagiques [2].

Si, dans la plupart des cas, ces levains sont utilisés avec satisfaction, ils pourraient probablement encore être améliorés par l'élimination des clones ayant des défauts majeurs. Cela permettrait l'élaboration de levains multiples [8], plus intéressants que plusieurs ferments utilisés en rotation ; cela aurait le double avantage de limiter les repiquages et d'entraîner une plus grande stabilité des fabrications successives.

Les faits invoqués ci-dessus sont transposables à la connaissance de la flore du lait cru, dans la mesure où il peut être établi que l'acide nalidixique qui était, dans ce travail, le facteur de sélection des germes gram-positifs, n'exerce aucune action inhibitrice, totale ou partielle, directe ou indirecte, sur les streptocoques et lactobacilles.

\section{Ré s u m é}

Les levains naturels préparés dans quatre laiteries de Normandie, à partir de lait cru par inhibition des bactéries Gram-négatives à l'aide de l'acide nalidixique, sont presque uniquement formés de streptocoques. Streptococcus lactis y domine (72 p. 100), suivi de S. diacetylactis. Par la méthode adansonienne, fondée sur les coefficients de similitude de ces deux espèces, on peut mettre en évidence six taxons.

Le pouvoir acidifiant et la faculté de multiplication sur lait UHT à $30^{\circ} \mathrm{C}$ en $8 \mathrm{~h}$ varient considérablement d'un clone à l'autre. Les polyrésistants aux antibiotiques existent en assez petit nombre.

\section{SUMMARY}

STUDY OF NATURAL STARTERS PREPARED IN NORMANDY DAIRY PLANTS BY INHIBITION OF THE GRAM-NEGATIVE BACTERIA

The natural starters prepared from raw milk in four Normandy dairy plants by inhibition of the Gram-negative bacteria with nalidixic acid are quite entirely constituted by streptococcus (S. lactis 72 p. 100), $S$. diacetylactis $(26 \mathrm{p} .100)$. By the adansonnian method, based on the similarity coefficient between the two species, it is possible to distinguish six taxons. The acidification power and the faculty of multification are considerably, varying from one strain to another. The antibiopolyresistant Streptococcus lactis are existing in few number. 


\section{Bibliographie}

[1] Adanson (M.) (1963). - Famille naturelle des plantes. Un vol., Vincent Ed., Paris.

[2] Degle (I.) et Busse (M.) (1978). - Bactériophages dans les levains lactiques traditionnels. C.R. $20^{\mathrm{e}}$ Congrès International de Laiterie, 568.

[3] Desfleurs (M.) et Desfleurs (M.) (1977). - Evaluation de la teneur en germe Gram négatifs des laits et ses conséquences. Le Lait, 57, 375-383.

[4] Felmingham (D.) et Juffs (H.) (1978). - Conservation par congélation des échantillons de lait cru pour analyse microbiologique. C.R. 20 Congrès International de Laiterie, 184.

[5] HoGg Mc (C.) and JAGo (G.) (1970). - Extraction of the $260 \mathrm{~nm}$ absorbing material from group $\mathrm{N}$ Streptococci as a method for estimating cell growth. J. Dairy Res., 37, 199-202.

[6] Jacouet (J.) et Auxepaules (M.) (1978). - Le problème de la pollution du lait par les antibiotiques. Etat actuel de la question. Bull. Acad. Vét., 51, 73-79.

[7] Jaceuet (J.) et Thévenot (R.) (1961). - Le lait et le froid. Un vol., J. B. Baillière Ed., Paris.

[8] Limsowtin (G.), Heap (H.) et Lawrence (R.) (1978). - Concept du levain multiple pour la fabrication du fromage. C.R. $20^{\mathrm{e}}$ Congrès International de Laiterie, 570.

[9] de Man (J.), Rogosa (M.) and Sharpe (M.) (1960). - A medium for the cultivation of lactobacilli. J. Appl. bact., 23, 130-135.

[10] MaRtley (F.) (1975). - Comportement et rôle des streptocoques lactiques du levain en fabrication de Camembert. Le Lait, 55, 310-323.

[11] Paule (R.) (1971). - Contribution à l'étude biochimique du genre Lactobacillus par une méthode normalisée (1971). Thèse Doctorat en pharmacie, Lyon.

[12] Reddy (M.), Veidamuthu (E.), Washam (C.) and Reinhold (J.) (1969). - Differential agar medium for separation S. lactis and S. cremoris. Appl. microbiol., 18, 755-759. 